\title{
Políticas de conservación y producción agropecuaria. ¿Cómo se integran los sistemas socioproductivos de Paso Centurión y Sierra de Ríos? Un abordaje desde la integralidad universitaria ${ }^{1}$
}

\author{
Daniel González Fajardo, ${ }^{2}$ Mariana Quintana, ${ }^{3}$ \\ Pablo Capurro, ${ }^{4}$ Lucia Greco Castro ${ }^{5}$
}

Recibido: 21/04/2020; Aceptado: 26/10/2020

DOI: https://doi.org/10.37125/ISR.7.1.6

\section{Resumen}

El presente trabajo es producto de las prácticas desarrolladas en Paso Centurión y Sierra de Ríos, departamento de Cerro Largo, por un grupo interdisciplinario que comprende las facultades de Ciencias, Ciencias Sociales, y Psicología, a través de distintos proyectos financiados por la Universidad de la República. El objetivo del equipo en territorio consistió en analizar, por un lado, los procesos de construcción de territorialidad en esta Área Protegida del Uruguay (Sistema Nacional de Áreas Protegidas), y por otro identificar tensiones entre los sistemas productivos y los sistemas de conservación ambiental. Para ello, se analizaron las pautas orientadas a la creación de un plan de manejo para el área, y se contrastó con la información de los sistemas socioproductivos de la zona. En los últimos 15 años Uruguay ha cambiado su producción agropecuaria propiciando un gran crecimiento del rubro forestal, en contraposición con la histórica tradición ganadera, así como también se han introducido cambios en relación con la conservación de áreas, a los cuales el departamento de Cerro Largo no ha sido ajeno. Los resultados obtenidos se analizaron según la categorización poblacional de las ruralidades uruguayas, identificando tensiones entre empresarios, familias productoras

1 Este artículo surge de la sistematización de los proyectos de investigación (PAIE) y extensión (PEUU) y Actividades en el Medio, realizados por el Grupo de Estudios Territoriales y Asociativismo Rural (GETAR) desde el 2016 al 2019, en Paso Centurión, Cerro Largo.

2 Instituto de Psicología Social, Facultad de Psicología, Universidad de la República (Udelar). dangonfaj@gmail.com

3 Licenciatura en Psicología, Facultad de Psicología, Udelar. emebutaca@gmail.com

4 Licenciatura en Geografía, Facultad de Ciencias, Udelar. pacaqui@gmail.com

5 Licenciatura en Trabajo Social, Facultad de Ciencias Sociales, Udelar. luciagrecocastro@gmail.com 
y asalariados rurales, con respecto a las dimensiones sociales, productivas, ambientales y económicas. La información relevada se obtuvo a través de: entrevistas a referentes de la zona, puesta de manifiesto del proyecto (instancias públicas), relevamientos en bases de datos de organismos públicos y estudios sobre sistemas socioproductivos en Uruguay. El resultado nos muestra un nudo principal de conflicto en la zona, vinculado a la producción forestal del sector empresarial y a la producción familiar ganadera. De la disputa de estas territorialidades surge el proceso de ingreso de Paso Centurión a los sistemas de conservación ambiental departamentales y nacionales.

Palabras clave: sistemas productivos; áreas protegidas del Uruguay; sustentabilidad; población rural; integralidad

\section{Un acercamiento a Paso Centurión}

El presente equipo de trabajo está integrado por estudiantes y egresados de la Universidad de la República de las licenciaturas en Psicología, Geografía y Trabajo Social. Como equipo venimos trabajando en la zona de Paso Centurión y Sierra de Ríos desde el año 2015 a través de diversos proyectos de investigación y extensión universitaria, para consolidar un Grupo de Estudios Territoriales y Asociativismo Rural (GETAR). En este tiempo nos hemos dedicado a fortalecer las organizaciones locales de asociativismo e interesados en especial en abordar el escenario generado por el ingreso del área al Sistema Nacional de Áreas Protegidas (SNAP). Este primer apartado busca introducir brevemente las características del lugar y su proceso de ingreso al SNAP.

Paso Centurión es un pequeño poblado ubicado a $60 \mathrm{~km}$ de la capital departamental de Cerro Largo, en dirección Este, limítrofe con Brasil. Esta zona, tradicionalmente ganadera, ha transformado su uso productivo del suelo generando un gran avance del uso forestal de la tierra, a través de grandes capitales que han adquirido campos en los últimos años. Este cambio del uso del suelo, no es una realidad ajena al resto del país, dado que el crecimiento en los últimos diez años a nivel nacional ha sido exponencial, amparado en marcos legales que permiten declarar suelos de prioridad forestal (Uruguay, 1987) y gobiernos que han estimulado las inversiones extranjeras directas. En la actualidad, en Uruguay existen dos grandes papeleras de pasta de celulosa (principal destino de la producción forestal) y se está en negociaciones por la instalación de una tercera muy cerca de nuestro campo de investigación.

Junto con el avance del modelo agroindustrial, se ha creado en el año 2000 y puesto en marcha desde 2005 (Uruguay, 2000) un Sistema Nacional de Áreas Protegidas, con el cual se espera definir áreas de conservación con alto valor ecosistémico. En la actualidad, hay Áreas Protegidas (AP) de distintos tipos de administración (público o privado) y con diversas categorías (sitios de protección, áreas de manejo de hábitats o especies, área protegida con recursos manejados, parques nacionales, paisajes protegidos y monumentos naturales). 
La zona de Paso Centurión y Sierra de Ríos fue declarada reserva departamental por la Intendencia de Cerro Largo (ICL) y luego Área Protegida Nacional en 2019. El estilo de vida de la comunidad genera movilizaciones de sus pobladores y pobladoras contra el avance forestal con la finalidad de conservar el valor paisajístico y la producción familiar. Esta población ha vivido a base de producción ganadera y esa práctica ha permitido los estados de conservación que se pueden encontrar en este sitio. De todas maneras, la producción forestal sigue creciendo y generó un campo de disputa territorial con los habitantes que han logrado mantenerse en la zona. Esto produce movimientos a nivel de la comunidad que se transforman en organizaciones de distinto tipo, en favor o en contra del área protegida. Respecto a las agrupaciones que se posicionan a favor del ingreso al SNAP podemos identificar aquellas vinculadas a proyectos emergentes de turismo, pequeños propietarios y arrendatarios de tierras, emprendimientos asociativos de producción ganadera, instituciones públicas y ONG vinculadas a la conservación ambiental. La producción familiar (de pequeña y mediana escala) no cuenta con márgenes amplios de inversión y necesita de ciertas condiciones para lograr la estabilidad. Por otra parte, encontramos a quienes rechazan el ingreso de la zona al SNAP. Estos son principalmente grandes propietarios y algunos actores políticos locales que defienden el avance de la industria forestal en la zona, dado que el aumento de la actividad (en Cerro Largo y en Uruguay) generó un importante incremento en los precios de la tierra (Garay et al., 2019). De esta manera se han beneficiado económicamente los propietarios de grandes extensiones de tierra que tienen capacidad de negociación con las empresas forestales. Esta tensión por el choque de construcciones territoriales, resulta de especial interés, el caso de Paso Centurión y Sierra de los Ríos como un territorio en transformación y disputa, que involucra a la comunidad, el sector empresarial y al Estado a través del SNAP. Una vez que fue declarada el área protegida, el SNAP tendrá que comenzar a trabajar en la creación de un plan de manejo que impondrá las condiciones de uso del área. Este proceso prevé espacios consultivos con la población y una Comisión Asesora Específica (CAE), pero no son vinculantes, dado que las condiciones de uso son definidas por criterios técnicos. Aun así, es de destacar que, si bien los planes de manejo se definen por criterios técnicos, existen antecedentes en esta comunidad que demuestran la posibilidad de generar modificaciones en las políticas de conservación a través de la organización (Santos, 2011). Por tanto, interesa a esta investigación observar cómo se produce la integración de los sistemas productivos a los sistemas de conservación y cómo puede incidir la instalación de un plan de manejo.

\section{La construcción del plan de manejo: la institucionalidad en clave territorial}

Encarna Herrera (1994) define el uso del concepto de integración en ciencias sociales como aquella interacción entre prácticas singulares y las normativas de un país. Este 
concepto permite pensar a la integración como: la interacción entre prácticas de instituciones públicas y prácticas privadas.

En un contexto de ingreso de la zona al sNAP parece conveniente poder relevar los sistemas socioproductivos de la región analizando su potencialidad para la implementación de políticas de conservación. En este sentido entendemos como Humberto Tommasino y Jorge Marzaroli (2008) que la sustentabilidad significa

... permanencia en el tiempo de formas de producción familiar con niveles aceptables de calidad de vida y beneficio económico. Esta perdurabilidad implica que el sistema de producción garantice el cuidado de ciertos aspectos de la naturaleza, permitiendo que las siguientes generaciones puedan mantener una forma de vida determinada y que esté garantizada la distribución equitativa -inter e intrageneracional- de los beneficios sociales, económicos y ecológicos (p. 24).

En cuanto a la caracterización ocupacional, la mayoría de los habitantes se dividen en dos rubros; el sector agropecuario y el sector de los servicios (manufactureros, comerciantes, empleados públicos, etc.). En este trabajo hemos optado por adoptar la categoría socioproductivo, teniendo en cuenta que las propias características de las ruralidades uruguayas, hacen que el trabajo y lo sociodemográfico sean casi indivisibles a la hora de caracterizar las distintas poblaciones rurales. Desde la perspectiva teórica del campo uruguayo, existe una abundante caracterización de los sistemas socioproductivos, que aplican al objetivo de caracterizar el área abordada. En este sentido utilizamos los aportes de Marta Chiappe, Matías Carámbula y Emilio Fernández (2008), como modelo para la caracterización. En este trabajo se definen tres categorías: asalariados, productores familiares y empresarios rurales.

En la tipificación de Chiappe, la principal característica de los empresarios rurales (Chiappe, Carámbula y Fernández, 2008, p. 75) es que explotan su producción fundamentalmente a través del empleo de mano de obra asalariada, participen en la producción o no, el trabajo asalariado siempre es predominante. Dentro de estos, los empresarios ganaderos se caracterizan por priorizar la posesión de la tierra frente a la inversión, con uso extensivo de este recurso y sistemas tradicionales de explotación con escasa incorporación de mejoras.

Por otra parte, el productor familiar se entiende «como aquel productor pobre, con pocos recursos productivos (poca tierra, maquinaria escasa y vieja, poco capital) y bastante tradicional (reticente a la adopción de tecnología)» (Chiappe, Carámbula y Fernández, 2008, p. 83). Así, predomina el trabajo familiar sobre la tierra (en propiedad o en usufructo). Mientras que parte de la producción se destina al autoconsumo, la mayoría se vuelca en mercados formales, distinguiéndose por esta característica del campesinado.

En cambio, el asalariado rural es concebido como «todos aquellos trabajadores que lo hacen por un salario sea este en forma mensualizada, jornalizada, a destajo o según cualquier forma de pago» (Chiappe, Carámbula y Fernández, 2008, p. 103). En 
cuanto a la relación laboral según rubros productivos, la relación laboral permanente se da más en la ganadería, mientras que en el rubro forestación es de tipo temporal.

Esta investigación propone análisis reflexivos de la integración de sistemas socioproductivos, sobre todo con base en la necesidad de la construcción de un plan de manejo coherente con la actividad productiva de las personas de la zona y con una propuesta de conservación que no afecte las condiciones necesarias para la producción familiar (sustentabilidad). ¿Por qué nos referimos a los planes de manejo? Los planes de manejo son reglamentos que, además de caracterizar la zona y describir las especies prioritarias para la conservación, condicionan las actividades que pueden hacerse en las áreas protegidas. Teniendo en cuenta los antecedentes bibliográficos de implementación de planes de manejo, en otras áreas protegidas también se han generado tensiones entre las personas que habitan el lugar, las empresas y el Estado con su perspectiva de desarrollo y ordenamiento territorial (Santos, 2011; Fernández, 2018; Santos y Chouhy, 2018). Algunos trabajos referentes para el equipo en términos de áreas protegidas, como el de Garay et al., (2019), dan cuenta a su vez de la conflictividad en las relaciones con la implementación del SNAP, teniendo como referencia también los aportes de Naína Pierri y Guillermo Foladori (2005) acerca de las contradicciones de las políticas de desarrollo sustentable llevadas por el Estado en los últimos 15 años. Por este motivo reafirmamos la necesidad de identificar tensiones potenciales entre los sistemas productivos actuales de Paso Centurión y Sierra de Ríos y las políticas de conservación que puedan implementarse con la consolidación de un plan de manejo para el área. Este proyecto se fundamenta en una fuerte pertinencia social para los pobladores, dado que la implementación de un sistema de conservación de la biodiversidad, puede entrar en conflicto con los sistemas productivos que se desarrollan en la zona. Este proyecto tuvo como finalidad que los resultados sean útiles en la construcción de un plan de manejo representativo para el área y sus habitantes. Para lograr ese objetivo problematizamos la información con la población local, a través de entrevistas y talleres, acerca del proceso de construcción del plan de manejo y el análisis de potenciales tensiones, así como también se hicieron observaciones participantes en las cuales robustecieron algunas conclusiones. A su vez, se generó un registro organizado de las principales actividades productivas con base en datos de organismos públicos, las cuales fueron contrastadas a la luz de las pautas preliminares recomendadas por el SNAP para el plan de manejo. Esta información fue analizada en conjunto con la población, a través de entrevistas en los hogares y actividades con grupos de la zona, entre ellos emprendimientos turísticos, emprendimientos de producción asociativa, escuelas y ong. De esta manera se generó un proceso de estudio que incorpora a la población local en el análisis crítico de la implementación de esta política, teniendo en cuenta a su vez el potencial organizativo preexistente. 
En relación con el marco nacional, consideramos que este trabajo es un aporte a la implementación de políticas territoriales de conservación y a la integración de los sistemas socioproductivos en los sistemas de protección de la biodiversidad.

\section{Investigar con y para los otros}

Tanto la estrategia metodológica, como la elección de la temática abordada, son producto de un largo proceso de trabajo con la población local. Este acercamiento surge del interés de estudiar las políticas de conservación ambiental, el asociativismo como estrategia para la organización comunitaria y la construcción de territorialidad en las ruralidades uruguayas, entendidas como apropiación y subjetivación de prácticas y espacios sociales (Haesbaert, 2004).

La articulación de experiencias y realidades que interactúan en el trabajo con las comunidades, lo que entendemos por diálogo de saberes. Este concepto expresa la dimensión dialógica de los procesos que se realizan en los territorios, lo que implica el reconocimiento de la validez de los conocimientos que poseen los sujetos, desde la población local; en conjunto con el accionar de las instituciones (Ghiso, 2000). Esta propuesta desde las metodologías participativas de educación popular en la crítica a la división positivista sujeto-objeto que promueve un abordaje objetivo hacia la realidad social. El diálogo de saberes refiere a la relación sujeto-sujeto, que es política porque apuesta la transformación de las desiguales condiciones de vida existentes; donde los diferentes saberes son complementarios y están en continuo diálogo.

En esta línea, esta investigación retoma los principios de integralidad promovidos desde la Universidad de la República a partir de la segunda reforma universitaria (Universidad de la República, Rectorado, 2010), donde se articulan las funciones de enseñanza, investigación y extensión (Arocena et al., 2011). Por tanto, la investigación no se escinde de los procesos dialógicos generados desde las propuestas de extensión, ni de la generación de aprendizajes. Estas temáticas han acompañado lo trabajado en el territorio a partir de 2015 y de allí en más hemos continuado estrechando estos vínculos entre conocimientos locales y académicos. Específicamente para esta investigación se llevaron a cabo entrevistas y talleres individuales y colectivos en torno a la temática AP, donde logramos identificar y comparar las diferentes perspectivas, dificultades y tensiones que subyacen al área.

En relación con la estrategia utilizada para la recolección de datos para esta investigación, relevaron unidades productivas con datos del Instituto Nacional de Estadística (INE) y de la Dirección General de Desarrollo Rural (DGDR) del Ministerio de Ganadería, Agricultura y Pesca (MGAP). Con esto se seleccionaron familias productoras, asalariados/as e informantes calificados, los cuales fueron entrevistados acerca del proceso de implementación del AP y de los sistemas productivos de la zona. Las dimensiones para el análisis son de carácter descriptivo y se construyeron a base de las conceptualizaciones teóricas existentes y tomando como referencia, el modelo 
de evaluación de sistemas productivos a través de los indicadores de sustentabilidad propuestos por Tommasino y Marzaroli (2008). Esta etapa fue acompañada de recorridas en el área que permitieron conocer las zonas donde la producción forestal es predominante. Para establecer este recorrido se utilizaron los relevamientos de población y producción mencionados, y se logró caracterizar su distribución productiva y poblacional. Asimismo, se trabajó sobre los datos existentes y se generaron cartografías que permitieron contrastar los volúmenes de cada tipo de producción (ganadera o forestal). A tales efectos se trabajó sobre datos de las actividades productivas, extraídos de distintas bases de datos de instituciones estatales, ${ }^{6}$ de acceso libre, en los últimos veinte años: población (INE), recursos naturales (MGAP, Ministerio de Vivienda Ordenamiento Territorial y Medio Ambiente [мvотма]) y otras posibles variables cuantitativas que permitieron elaborar cartografías socioproductivas hacia la conformación de un perfil socioterritorial de la zona de estudio. La síntesis entre estos datos y las observaciones de campo, habilitaron un acercamiento a los sistemas socioproductivos de Centurión y su aspecto prospectivo respecto al Área Protegida y los planes de manejo. La elaboración de las cartografías se realizó fundamentalmente con sistemas de información geográfica de uso libre (GVSIG, QGIS), con base en datos espaciales en formato shp.

Principalmente utilizamos como técnicas de abordaje de trabajo de campo las entrevistas abiertas (Valles, 2002) y los talleres (Cano, 2012), buscando sistematizar las distintas opiniones que refieren al área. Estas entrevistas fueron realizadas a aquellos pobladores que, por sus características de ubicación geográfica, formas productivas o de vida, resultan necesarios para un análisis más profundo.

Otra de las formas de recolección de datos fue, como hemos mencionado, a partir de la observación participante (Valles, 2000) en audiencias públicas del sNAP. Esta instancia ocurrió en el mes de abril de 2018 en dos jornadas (una en Melo y otra en Paso Centurión). Esto permitió tener una observación de los grupos (a favor y contra el ingreso); de las instituciones públicas (tanto estatales como departamentales) y los técnicos y profesionales que desarrollan prácticas en la zona. Estas sesiones fueron grabadas con la autorización del SNAP y utilizadas únicamente para el desarrollo de esta investigación. Para robustecer el análisis de la postura de cada grupo mencionado se consultaron artículos de prensa (columnas de opinión y producción periodística), sobre los acontecimientos que se estaban suscitando en la zona y tomados como material secundario de la investigación.

Como hemos mencionado al principio de este apartado, nuestro trabajo en la zona está fuertemente implicado en la construcción de lazos recíprocos entre las comunidades organizadas y la universidad. Esto nos lleva a generar procesos más largos que los contemplados por los proyectos universitarios, ya que son dos lógicas temporales disociadas. Por tanto, desde el equipo de trabajo optamos por incluir tanto los

6 Las bases consultadas corresponden al MGAP, a la Dirección Nacional de Minería y Geología, al Ministerio de Transporte y Obras Públicas, al Ministerio de Industria, Energía y Minería y al sNAP. 
conocimientos y experiencias previas a la ejecución del proyecto como las instancias de intercambio de los resultados con la comunidad. Estas últimas, a su vez, aportan nuevo material de análisis, que nos compromete a continuar trabajando en el estudio de las disputas territoriales, en las ruralidades uruguayas. Consideramos que las comunidades organizadas tienen mayores posibilidades de aportar a la construcción de políticas, que apunten a la sustentabilidad de la producción familiar y para lo cual la universidad debe acompañar con prácticas integrales.

\section{Caracterización de los sistemas socioproductivos}

\section{Los empresarios rurales}

Dentro de esta categoría se encuentran las empresas forestales, que ocupan más de siete mil hectáreas del AP y sus principales representantes son Forestal Oriental y Montes del Plata (Oyhantçabal y Narbondo, 2016). Por las formas de producción de este rubro, existe poca mano de obra asalariada y gran inversión tecnológica en cuanto a maquinaria, logística, genética e insumos. Estas empresas manifiestan su descontento con la implementación del área, argumentando que compraron campos y contaron con proyectos aprobados por la Dirección Nacional de Medio Ambiente (Dinama) del mvotma para esta actividad productiva. Sin embargo, con la implementación del AP, se prohibirá el desarrollo de esta actividad. Se toma como una contradicción el hecho de que los suelos sean declarados de prioridad forestal y luego restringidos en su uso por medidas de conservación.

Asimismo, los empresarios ganaderos poseen emprendimientos de grandes extensiones vinculados a la producción, sobre todo, de bovinos. Hemos constatado una retroalimentación entre estos emprendimientos con los forestales, que permiten el avance rápido del rubro en la zona, a través de la capacidad de adopción tecnológica y la concentración de la tierra. Al ser propietarios de grandes extensiones de campo se fomentan las medianerías con forestales e inclusive el silvopastoreo (combinación de ganadería y producción forestal en el mismo predio). Esto genera un alza en los precios de la tierra tanto para arrendamientos como en ventas. Con estas posibilidades de producción, el sector empresarial no reside permanentemente en la zona, sino que cuenta con mano de obra asalariada, que está a cargo de las tareas cotidianas y en general reside en dichos campos.

Encontramos a su vez otras empresas, de menor porte, que representan otros modelos productivos empresariales vinculados con la agroindustria arrocera, la minería y parques eólicos, muchos vinculados a las mismas familias ganaderas empresariales. Una de las tensiones que se pudieron observar en la puesta de manifiesto del ingreso del área, se vincula con los parques eólicos. En este caso el sNAP negoció la creación de un área adyacente, para que el parque eólico ya instalado en la zona no fuera incluido dentro de las restricciones para el área. 
Esta alianza entre las empresas de la zona que complementan recursos y producciones encuentra un límite en la instalación del área protegida y por ello han organizado un grupo contra la instalación del AP. Algunos defensores de la producción forestal en la zona son Ignacio Gigena (edil del Partido Nacional) y Goy Viera, ambos empresarios rurales que pertenecen a un grupo de productores que se ha manifestado en contra del Ap, que ven afectado su patrimonio.

Algunos de los argumentos esgrimidos por el grupo están asociados a que: «En esos campos "es muy difícil" realizar ganadería o agricultura y "la forestación se convirtió en una salvación” para el productor...» (Búsqueda, 2018). A su vez afirman que el alza del precio de la tierra fue un beneficio para los propietarios y ahora ven con preocupación la posible pérdida de valor de los campos en el mercado. Otros argumentos manejados por este grupo y algunas empresas forestales como POSCO (Garay et al., 2019), es que la forestación no afecta la biodiversidad: «¿De dónde sacó la versión de que los eucaliptos perjudican la biodiversidad cuando es exactamente lo contrario?» (Viera, 2018). Un tercer discurso que se propone desde este grupo es que de fondo habría una cuestión «ideológica», «una venganza» del gobierno progresista sobre el sector de los empresarios rurales. «Sin dudas, existe el consenso nacional de que debemos proteger el medioambiente y la biodiversidad con arreglo a las necesidades ecológicas del territorio nacional. Pero, no se debe usar esa circunstancia como instrumento de aplicación de una venganza ideológica» (Viera, 2018). A su vez estos últimos argumentos se conjugan en cuanto a los efectos de la producción:

Está bien que se proteja al monte indígena y su biodiversidad, pero está mal que se afecte a los campos adyacentes tradicionalmente ganaderos que, nunca perjudicaron ni lo van a hacer, y además ayudan al ecosistema a través de verdeos y praderas artificiales (Viera, 2018).

Muchos de los grandes propietarios se sienten afectados por la medida, ya que pierden valor de negocio y posibilidad de explotación intensiva de la tierra.

\section{Producción familiar}

Este sector involucra a la mayoría de la población permanente de Paso Centurión y Sierra de Ríos. Según datos de la DGDR (2018) hay 287 personas vinculadas a esta modalidad socioproductiva, en relación con los datos del INE de 2011, podemos afirmar que su representatividad en el territorio en cuanto a población permanente es casi del $100 \%$. Casi el total de los emprendimientos está vinculado a la ganadería (ovina y bovina) y existen dos campos del Instituto Nacional de Colonización (INC) gestionados de forma grupal. La población permanente, continúa una tendencia de paulatina disminución de representatividad en la zona, donde la mayor concentración se encuentra al norte del AP (Centro Poblado de Paso Centurión), diferenciándose de los lugares donde el avance forestal ha sido más significativo (este y sur del área). 
Como observamos en la Figura 1 y en las Tablas 1 y 2 la mayoría de la población se dedican a otras actividades no relacionadas con la actividad forestal.

La representatividad territorial de la producción familiar está en competencia directa con la forestación. Ambas son las formas más comunes de producción, ocupando en conjunto un $86,7 \%$ de la superficie del AP.

\section{Figura 1.}

Predios relevados a 2018 en el Registro de Productores/as Familiares (DGDR-MGAP)

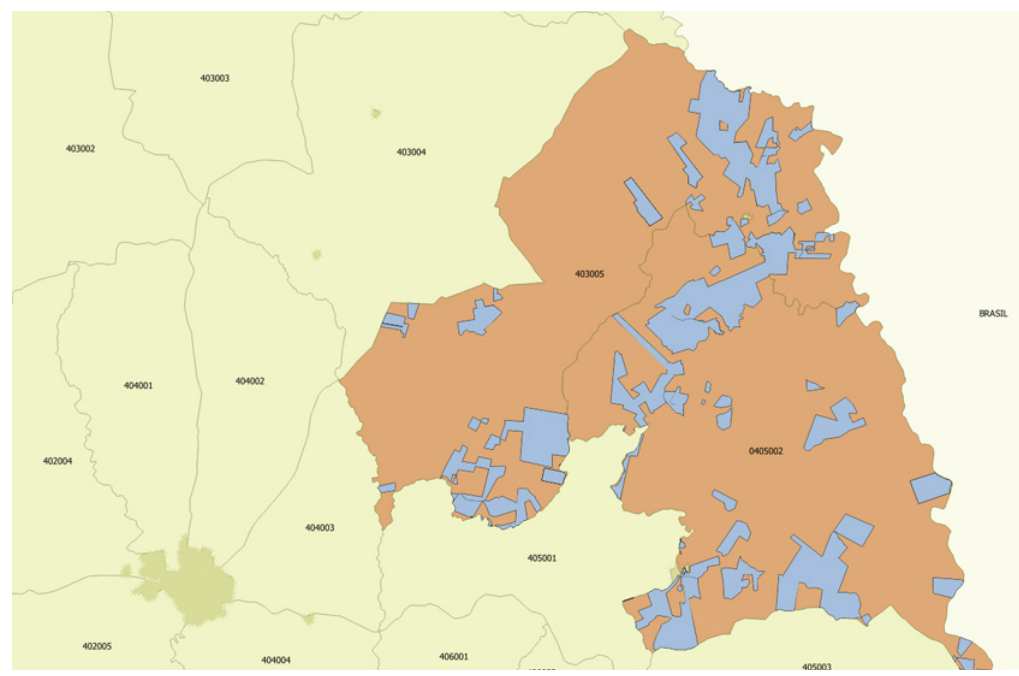

Fuente: Registro de Productores/as Familiares (DGDR, 2018).

\section{Tabla 1. Características de las familias}

\begin{tabular}{l|r}
\hline $\begin{array}{l}\text { Total de personas vinculadas a las Unidades } \\
\text { de Producción Familiar }\end{array}$ & 287 \\
\hline Mujeres (total) & 133 \\
\hline Varones (total) & 154 \\
\hline
\end{tabular}

Fuente: Registro de Productores/as Familiares (DGDR, 2018) 
Tabla 2.Unidades Productivas (UPF) registradas en la zona.

Distribución por rubro productivo principal

\begin{tabular}{l|r}
\hline Rubro principal & Unidades Productivas Familiares \\
\hline Agricultura de cereales y oleaginosos & 1 \\
\hline Aves & 1 \\
\hline Ganadería de carne & 124 \\
\hline Ganadería de lanA & 3 \\
\hline Total & 129 \\
\hline
\end{tabular}

Fuente: Registro de Productores/as Familiares (DGDR, 2018).

\section{Asalariados}

La mayoría de los asalariados están vinculados a los empresarios ganaderos. Estas personas residen en campos de sus patrones o trabajan en otros campos en actividades relacionadas a la ganadería (alambradores, esquilas, carpintería rural, caza de animales considerados plagas, etc.). Asimismo, estos asalariados consiguen permiso de pastoreo de los empresarios ganaderos y así complementan sus ingresos. Se debe tener en cuenta que las categorías de producción familiar y asalariado/a se yuxtaponen en las personas que viven allí, que pertenecen indistintamente a una categoría u otra.

\section{La creación del plan de manejo}

Actualmente existen cuatro planes de manejo de AP en Uruguay: Valle del Lunarejo (2016), Esteros de Farrapos (2014), Quebrada de los Cuervos (2010) y Laguna de Rocha (2016). Para el estudio de los posibles impactos de la implementación de un plan de manejo, para la zona de Paso Centurión y Sierra de Ríos, se hizo un esquema comparativo de los planes de manejo creados por el sNAP.7 Aquí nos encontramos con la dificultad de que cada plan de manejo utiliza distintas categorías de actividades según las características propias del territorio y sus necesidades de conservación. De esta forma debimos construir nuevas categorías, más amplias, que permitan realizar un esquema comparativo de los planes de manejo existentes. Las categorías creadas fueron: Turismo y Deportes (incluye deportes acuáticos, pesca deportiva, frecuencia de turistas, infraestructura turística), Producción Ganadera, Producción Agrícola, Actividades Extractivas (tala de monte nativo, minería, forestación, pesca artesanal o industrial), Manejo de Campo (mejoramiento de campos), Aprovechamientos de Agua (pozos, azudes, diques), Infraestructura de Uso Agropecuario e Infraestructura Civil.

7 Los planes de manejo estudiados son de áreas de gestión estatal e integran el sNAP. Responden a las áreas protegidas de Laguna de Rocha, Valle del Lunarejo, Quebrada de los Cuervos y Esteros de Farrapos. 
A su vez estas categorías han sido ubicadas en niveles según el grado de intervención humana. En general las áreas dividen este grado de intervención en cuatro niveles: mínima, baja, media y alta, entre las que mínima indica las áreas que están más conservadas y Alta donde se ha modificado más fuertemente.

Para completar el análisis se construyeron gráficos comparativos (Figuras 2 y 3 ) de las áreas según los niveles de intervención y las categorías implicadas en los planes de manejo, la frecuencia fue codificada en cuatro variables que son: o (no corresponde), 1 (permitida), 2 (permitida con restricciones), 3 (no permitida).

Como se puede observar los gráficos son poco concentrados, con una dispersión oscilante entre las categorías no permitida y permitida con restricciones. Esto implica que en cualquiera de los grados de intervención existe algún tipo de regulación, así se explica que la moda del gráfico se ubique en permitida con restricciones y que sumado al conjunto con no permitida alcanzan casi el $65 \%$ de la frecuencia.

\section{Figura 2.}

\section{Esquema comparativo de actividades restringidas en los planes de manejo del SNAP para áreas de intervención mínima y baja}

Restricciones en áreas de intervención mínima

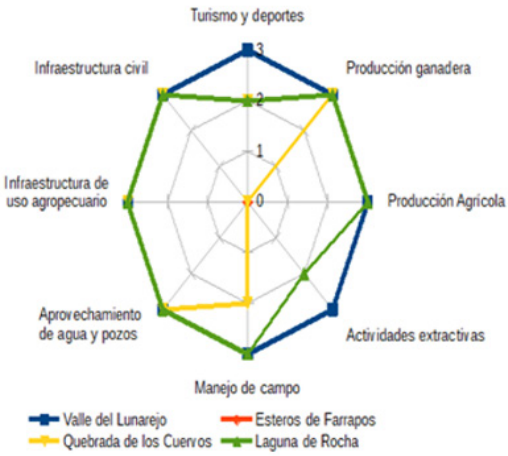

Restricciones en áreas de intervención baja

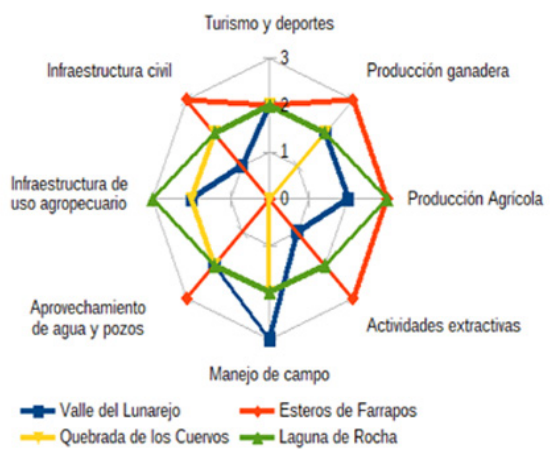

Fuente: elaboración propia a partir de datos de SNAP, 2011; SNAP, 2014; SNAP, 2016a; SNAP, 2016b 
Figura 3.

Esquema comparativo de actividades restringidas en los planes de manejo del SNAP para áreas de intervención media y alta

Restricciones en áreas de intervención media

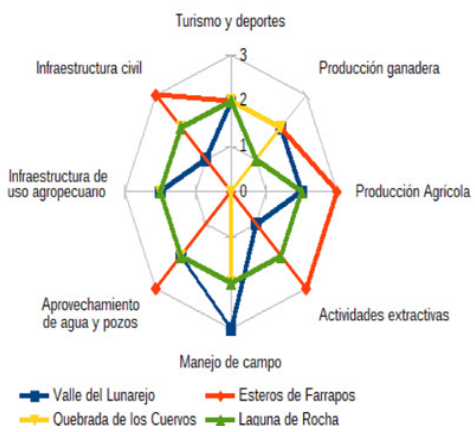

Restricciones en áreas de intervención alta

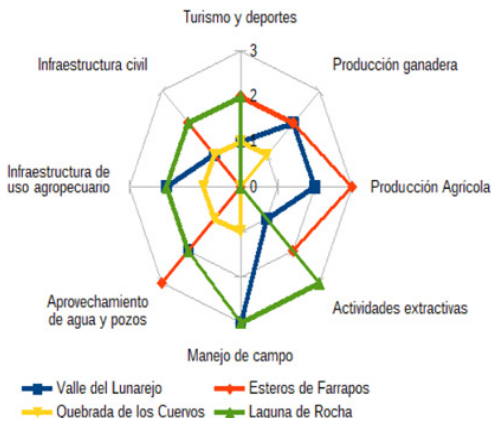

Fuente: elaboración propia a partir de datos de SNAP, 2011; SNAP, 2014; SNAP, 2016a; SNAP, 2016b

Este análisis nos permite afrontar de antemano la posibilidad de que (en caso de que corresponda) las actividades contempladas en el plan de manejo, exigirán al menos algunas restricciones para su desarrollo. Este insumo es de gran utilidad para abordar las recomendaciones del SNAP en cuanto a la implementación de un plan de manejo para el área.

\section{Entre lo prohibido y lo permitido con restricciones. Propuestas del SNAP para este plan de manejo}

En la propuesta de ingreso de Paso Centurión y Sierra de Ríos al SNAP, se proponen «Pautas para el plan de manejo y condiciones generales de uso» (SNAP, 2018). Esta propuesta recopila un conjunto de actividades a ser reguladas en cuanto al uso de los suelos y el agua en el área de influencia. Dicho documento afirma que la implementación del área no afectará los sistemas productivos relacionados a la ganadería. Sin embargo, para otros tipos de emprendimientos se proponen prohibiciones o restricciones. En este apartado nos proponemos identificar las posibles tensiones entre estas pautas y los emprendimientos existentes.

\section{Procesos de urbanización}

Los procesos de urbanización pueden afectar los corredores biológicos y comprometer los ecosistemas. Como hemos visto antes, en el resto de los planes de manejo existen antecedentes de restricciones en cuanto a obras de infraestructura civil. En principio este aspecto no debería ser una problemática, dado que el proceso de despoblamiento del área es muy acentuado y no existe mucha expectativa de que esto se revierta. 
En la actualidad, el principal centro poblacional es un núcleo de viviendas construidas con financiación estatal, donde viven unas diez familias. A lo largo del tiempo el territorio ha conservado un paisaje de poblado rural con distancias entre sus construcciones. En el proceso de trabajo que llevamos en el territorio, hemos podido recoger testimonios de vecinos y vecinas que hablan de una intención de volver a ser un poblado con cruce fronterizo hacia Brasil, lo cual generaría un mayor movimiento económico en la zona. La población cruza la frontera de manera informal, con botes o, cuando el río da paso, a caballo o a pie. En el pasado existía una aduana donde se tramitaban exportaciones de ganado a Brasil y luego, a principios del siglo xx, también existió una balsa para el cruce de la frontera. Esa idea de poblado fronterizo aún está presente en sus habitantes. Según el testimonio de vecinos y vecinas de la zona, existió un proyecto para construir un puente en esta zona, sobre el río Yaguarón, lo que sería un tercer cruce de frontera formal hacia el Brasil, junto con Aceguá y Río Branco. En la actualidad, esta idea volvió a tener algo de resonancia a partir del cambio de gobierno, pero no existe ningún proyecto formal.

\section{Monocultivo forestal}

La representatividad de la forestación en el AP es de más de siete mil hectáreas ubicadas en su mayoría en el área sur y sureste. En el mapa de la Figura 4 se muestra la distribución territorial de estos emprendimientos.

\section{Figura 4.}

Evolución de la producción forestal por sobre ecosistemas naturales
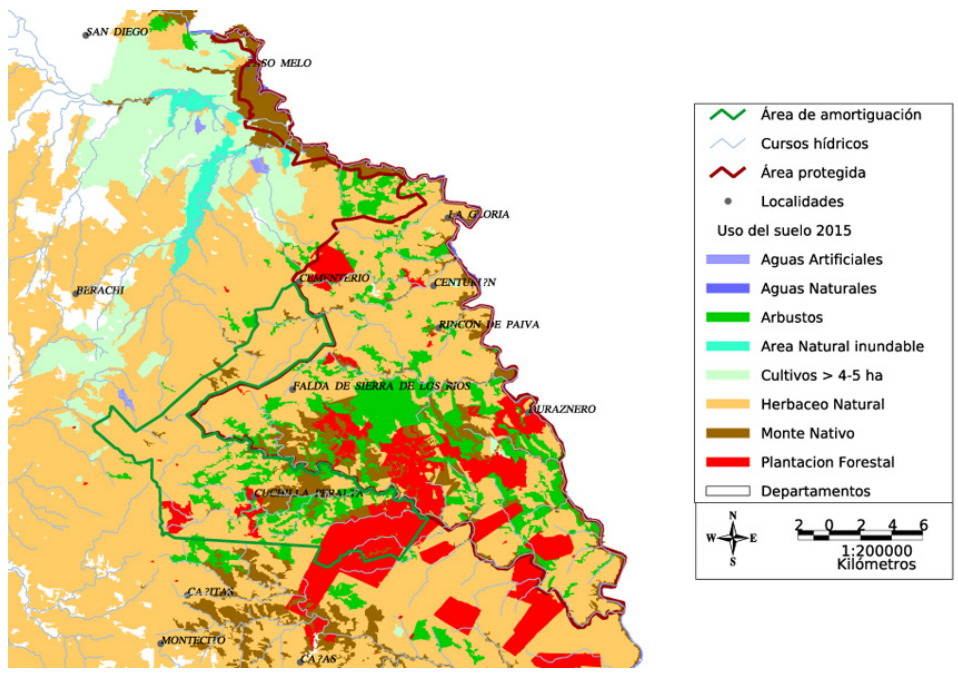

Fuente: elaboración propia a partir Dinama, 2015. 
Como se puede observar en la Figura 4, los emprendimientos forestales son uno de los más representativos en la zona y representan grandes extensiones que superan las cien hectáreas por emprendimiento. Las empresas con más emprendimientos en la zona son Lumin y Montes del Plata. Esta actividad ha crecido en los últimos diez años, generando un sacudón en el mercado de tierras (Garay et al., 2019). Según datos de la Dirección de Estadísticas Agropecuarias del MGAP el precio de la tierra en Cerro Largo en promedio pasó de USD 400 la hectárea en el año 2000 a USD 3100 la hectárea aproximadamente en el año 2016 (Garay et al., 2019). Esto fue confirmado por informantes calificados que constatan el aumento en las transacciones. De esta manera la inversión en tierras es una de las principales conflictividades entre empresas forestales y la implementación del AP.

Como hemos mencionado, y según el relato de vecinos y vecinas de la zona que vivieron el proceso del avance forestal, esta actividad requiere muy poca mano de obra. En una primera etapa de la producción se hacen los movimientos de tierra para luego comenzar el trasplante de los árboles. Debido a la alta tecnificación, las oportunidades laborales son escasas para los y las locales. La segunda etapa se refiere al proceso de crecimiento de los árboles que dura entre diez y quince años; durante este período la mano de obra es casi nula. Luego en la etapa de cosecha, aumenta el empleo, nuevamente la alta tecnificación hace que sean contratados trabajadores calificados para el uso de maquinaria específica. Lo mismo sucede en el transporte y la industria, se generan empleos a los que la población de la zona no logra acceder.

El proyecto SNAP propone medidas para regular los campos forestados, buscando retraer la actividad forestal de gran escala.

\section{Minería}

La presencia de explotaciones mineras es poco significativa en la zona. Existe una cantera autorizada por la Dinama que se sirve de los recursos minerales, para el mantenimiento de caminería en el acceso al parque eólico. A su vez otro de los puntos que suponen la explotación de recursos, son las perforaciones realizadas por ANCAP (aprobadas por la Dinama), para la exploración de yacimientos de hidrocarburos.

\section{Grandes infraestructuras (parques eólicos)}

Actualmente existe un solo parque eólico, que se ubica en la zona de Cuchilla de Peralta (zona adyacente del AP). Según entrevistas que hemos hecho a pobladores de Centurión, existen algunos empresarios de la zona, vinculados a la ganadería, que estarían trabajando por la futura instalación de otro parque eólico. Esta fue una de las discusiones que estuvieron en las jornadas, donde se puso de manifiesto la propuesta de ingreso de la zona al SNAP. El sNAP propone algunas recomendaciones para el plan de manejo del AP, donde dicen: «Los parques eólicos dentro del área adyacente no quedarán prohibidos...» (Sistema Nacional de Áreas Protegidas [SNAP], 2018). 
Según el informe de la Dinama:

El proyecto consiste en la instalación de un parque eólico dotado de hasta 25 aerogeneradores, totalizando una potencia máxima de 50 MW. [...] Durante la fase operativa no se esperan impactos significativos, siendo los más relevantes lo que pudieran afectar a la avifauna y al paisaje (Dinama, 2015).

\section{Tala de monte nativo}

La actividad de tala de monte nativo se utiliza de forma poco representativa. Existe su utilización para uso doméstico o para fabricación de piques asociados al alambrado de los campos. La presencia de monte nativo no representa un conflicto para la ganadería, la cual se sirve del recurso como abrigo/sombra para los animales. En la Figura 4 se muestra la distribución del monte nativo en el área y su competencia con la actividad forestal. La presencia más importante de monte nativo se puede observar en la ribera del río Yaguarón. El segundo lugar de concentración se encuentra en las zonas de quebradas, donde es casi imposible el desarrollo de actividades productivas.

\section{Introducción de especies exóticas y la caza}

En el tiempo de trabajo en la zona no se relevó específicamente la presencia de especies exóticas por fuera de la forestación. En las salidas de campo y los datos relevados por el SNAP en la propuesta de ingreso, se identificaron especies exóticas presentes, pero no representan invasiones significativas en el territorio.

Con respecto a la caza, se mantiene aquella que tiene fines de subsistencia y control de plagas, práctica común entre la población rural del Uruguay. En los últimos años se ha ido modificando la relación de la población local con la fauna nativa, y disminuyó la comercialización ilegal de estas especies (sobre todo aves). Consideramos que estos son los aspectos sobre los cuales existen menores discrepancias a nivel general, teniendo en cuenta el tratamiento especial que tiene la forestación (especies exóticas introducidas para la producción de madera) en las recomendaciones del SNAP para el plan de manejo.

\section{Algunas conclusiones acerca de la (des)integración de los sistemas productivos de Paso Centurión y Sierra de Ríos}

En lo que respecta a la integración de los sistemas socioproductivos en Paso Centurión, se puede establecer que existen modelos productivos que anteceden al AP y que logran la coexistencia con los ecosistemas. Sin embargo, estas modalidades productivas, se ven amenazadas ante el avance de modelos extractivos que generan mayores rentabilidades. De la colisión de estos dos modelos es que surge el AP, generando una confrontación de ideas entre las personas que desarrollan actividades en 
la zona, acerca de la conservación y el territorio. Por una parte, se pudo relevar una la alianza entre los empresarios forestales y los ganaderos, que han logrado dinamizar sus negocios con el ingreso de la producción forestal en la zona. Para este sector, el AP representa un retroceso al desarrollo de la zona. Por otra parte, la producción familiar ganadera generó sus alianzas con el Estado (tanto a nivel departamental, como nacional), con las cuales lograron mantener su espacio socioproductivo a través del SNAP.

El impacto del avance de la producción forestal produjo una reconfiguración territorial, dividiendo al área en dos grandes zonas socioproductivas: una predominantemente forestal y otra ganadera.

Hacia el sur y sureste del AP existe una mayor presencia de la producción forestal, con un alto precio de las tierras, que resulta en un proceso de aislamiento de la población y posterior migración de las/os pobladores. Por las características propias del modelo forestal, no se garantiza la creación de servicios que atiendan a las problemáticas básicas de la población, ni genera trabajo para esta. En la zona norte y noroeste del AP se identifica la mayor concentración de población y de producción familiar ganadera, la cual cuenta con modelos de producción de menor impacto sobre los ecosistemas. Además, sobre esta zona se han concentrado la población y los servicios. El AP para estas personas representa una herramienta para la sostenibilidad de sus proyectos y la permanencia en el territorio.

Evidentemente, desde las distintas políticas de estado, en las últimas tres décadas se ha fomentado con fuerza el desarrollo de la producción forestal en Uruguay. El tipo de clasificación Coneat (Comisión Nacional de Estudio Agroeconómico de la Tierra) es un instrumento que se ha utilizado para generar índices de productividad de la tierra y así de alguna manera valorizar los campos. Es entonces que el índice Coneat define (entre otros factores) el valor de la tierra. En Cerro Largo los índices de productividad de la tierra son bajos y por esto han sido declaradas en gran medida de prioridad forestal. Con esto se utilizan los suelos clasificados como menos productivos, para desarrollar actividades extractivas sin otra variable a tener en cuenta más que la productividad. A partir del fomento de la producción forestal la demanda de tierras en la zona aumenta y por tanto el precio de la tierra también. Esta gran demanda de tierras pone en cuestión la propia clasificación de productividad. Para la producción familiar de la zona que trabaja sobre suelos pocos productivos, muchas veces en arrendamiento, el aumento del precio de la tierra pone en riesgo su supervivencia. La herramienta del INC aporta a la sustentabilidad de estas poblaciones (en la zona hay dos campos colectivos), pero su representatividad en cantidad de hectáreas aún es insuficiente. A través del AP se espera que disminuya el precio de la tierra y esto genera conflictos de intereses entre los pobladores.

Para ser fieles a este proceso de instalación del AP en Paso Centurión y Sierra de Ríos, donde la mayoría de la comunidad fue demandando la conservación del paisaje, la regulación de los modelos productivos y de esta manera poder seguir viviendo allí; se debe construir un plan de manejo acorde a las necesidades de las familias 
productoras. En cada AP los procesos de instalación de los planes de manejo fueron distintos y, de hecho, la mayoría de las AP no cuentan aún con plan de manejo. Esto es una dificultad para las AP que han sido, como en Paso Centurión y Sierra de Ríos, creadas desde demandas locales para la solución de problemáticas de carácter nacional. Crear un plan de manejo que regule las actividades productivas en el territorio entra en contradicción directa con los instrumentos que fomentan la producción forestal, creando un territorio aislado de las lógicas productivas del resto del país. Este aspecto, como hemos visto, es parte del principal argumento que manejan los detractores del AP, ya que indican la contradicción de la idea de conservación por áreas. Desde este aspecto se puede entender que existen territorios a conservar y otros que son sacrificables por su escaso valor productivo o ecosistémico.

Por otra parte, observamos que desde el snAP se propone una construcción amplia del plan de manejo para poder contemplar las particularidades de cada territorio. Esto es positivo a los fines de contemplar los imponderables de cada territorio, pero genera la dificultad de poder comparar y sistematizar la regulación de las actividades productivas. Estas dificultades respecto al estudio de las regulaciones, impide la interconexión de las áreas, reproduciendo un modelo donde cada AP es un territorio con sus propias lógicas.

El Ap de Paso Centurión y Sierra de Ríos es el resultado de la iniciativa de la población para poder defender su permanencia en el territorio y el derecho a construir su territorialidad (Haesbaert, 2004). El principal nudo que identificamos en el territorio está vinculado a la forestación y el conflicto que esta genera sobre la producción ganadera. Los sistemas socioproductivos no se encuentran integrados entre sí, pese a los intentos de silvopastoreo, los territorios están definidos por su producción y sobre ellos se pueden ver las consecuencias de cada modelo. La producción ganadera ha generado desigualdades históricas sobre el acceso a la tierra, entre otros factores debido a la concentración de este bien en algunas familias, las pocas posibilidades de capitalización por parte de la producción familiar y las pocas posibilidades de inversión tecnológica. Estas desigualdades han favorecido a la concentración de la tierra y al ingreso de formas extractivas de producción, intensiva y a gran escala. Es así que el campo se encuentra en constante proceso de migración, debido a las pocas oportunidades que ofrecen las zonas de producción ganadera. Sin embargo, hemos sido testigos de que el avance forestal puede ser un factor que acelere aún más este proceso.

\section{Reflexiones en clave de integralidad}

A través de este recorrido pudimos generar prácticas de trabajo con una comunidad que se encontraba en disputas territoriales. Ese trabajo lleva unos cuatro años e implica una construcción conjunta entre población y universidad. La intervención en el territorio se centró en abordar las problemáticas que vivencian pobladores y pobladoras de la zona, en sintonía con la incorporación de una nueva política pública que 
busca conservar algunos ecosistemas. Desde nuestra perspectiva de trabajo hemos optado por respetar ciertas etapas de trabajo, que fueron desde diseños exploratorios de investigación a actividades de extensión. Todo este despliegue de propuestas de trabajo fue acompañado por las nociones de integralidad retomadas de la segunda reforma universitaria, donde investigación, educación y extensión forman parte de un mismo método de trabajo con la comunidad. Asimismo, integramos el equipo de forma interdisciplinaria, retomando principios de la geografía humana, la psicología social y la educación popular. Entendemos que los estudios territoriales implican poder transitar las relaciones que la comunidad genera con el tiempo, el espacio, el paisaje y los afectos. Eso lleva principalmente tiempo y en la actualidad los proyectos de trabajo tienden a ser a corto plazo.

Esta investigación nos permite pensar acerca de los procesos de ingreso de un territorio a un sistema de conservación ambiental y todos los mecanismos que se activan ante la instalación de estas políticas. Esta área protegida en particular se consolida a partir de una población que carece de herramientas de capitalización y una desigualdad histórica que no ha sido atendida, pero que sin embargo han generado espacios de participación donde se construyen espacios de gobernanza. Por tanto, para la universidad acompañar estos procesos implica poner a disposición el conocimiento científico al servicio de la comunidad.

Consideramos que una comunidad organizada tiene mayores posibilidades de construir áreas de conservación que reflejen sus realidades. La estrategia asociativa produce formas novedosas de territorialidad, que buscan superar las desigualdades históricas de las familias productoras y coincide con las necesidades de conservación ambiental. El presente trabajo busca potenciar experiencias que aporten a la construcción de territorios sustentables. Apostamos a que desde la Universidad de la República también se continúe apoyando estas experiencias y que adopte un rol más participativo en las contiendas por las disputas territoriales.

\section{Referencias}

Arocena, R., Tommasino, H., Rodríguez, N., Sutz, J., Álvarez, E., y Romano, A. (2011). Integralidad: tensiones y perspectivas. Cuadernos de extensión, (1). Montevideo: CSEAM, Universidad de la República.

Búsqueda (2018, noviembre 8). Productores arachanes aseguran que serán perjudicados por incluir sus campos en áreas protegidas, ya que no podrán forestar. Recuperado de https://www.busqueda. com.uy/Secciones/Productores-arachanes-aseguran-que-seran-perjudicados-por-incluir-suscampos-en-areas-protegidas-ya-que-no-podran-forestar-uc38516

CAno, A. (2012). La metodología de taller en los procesos de educación popular. Revista Latinoamericana de Metodología de las Ciencias Sociales, 2(2), 22-51. Recuperado de http://www.memoria.fahce. unlp.edu.ar/art_revistas/pr.5653/pr.5653.pdf.

Chiappe, M., Carámbula, M., y Fernández, E. (Comps.) (2008). El campo uruguayo: una mirada desde la sociología rural. Montevideo: Facultad de Agronomía-csic, Universidad de la República. 
Dirección General de Desarrollo Rural (DGDR) (2018). Registro de productores familiares. Información solicitada al MGAP. Recuperado de https://www. gub.uy/ministerio-ganaderia-agricultura-pesca/tramites-y-servicios/servicios/ registro-productores-familiares-agropecuarios

Dirección Nacional de Medio Ambiente (Dinama) (2015). Ficha Ambiental. Comunicación de proyecto. Parque Eólico Sarandí. Montevideo: mvotma.

FERnÁndez, A. (2018). Los procesos participativos en la planificación de áreas protegidas de Uruguay como oportunidad para mejorar la implementación de los planes de manejo. Tesis de Maestría en Manejo Costero Integrado del Cono Sur. Maldonado: cure, Universidad de la República.

Garay, A., Santos, C., Grattarola, F., Perazza, G., Taks, J., Bergós, L., ... Chouy, M. (2019). Informe final: Participación social, conocimiento experto y conflictos ambientales sobre uso del suelo, energía $y$ biodiversidad en Paso Centurión (Cerro Largo, Uruguay). Montevideo: Programa I+D, Csic, Universidad de la República, Montevideo.

Ghiso, A. (2000). Potenciando la diversidad. Diálogo de saberes, una práctica hermenéutica colectiva. Recuperado de http://parquedelavida.co/images/contenidos/el_parque/banco_de_conocimiento/ potenciando_la_diversidad.pdf.

Haesbaert, R. (2004) O mito da desterritorializaçao: do "fim dos territórios» á multiterritiralidade. Río de Janeiro: Bertrand Brasil.

Herrera, E. (1994). Reflexiones en torno al concepto de integración en la sociología de la inmigración. Papers. Revista de sociología. La construcción social del inmigrante, (43), 71-76. Recuperado de https://raco.cat/index.php/Papers/article/view/25209/58516.

Oyhantçabal, G., y Narbondo, I. (2016) ¿Acaparamiento de tierras en Uruguay? Evidencias en torno a las formas y la intensidad de la centralización de tierras en Uruguay. Precongreso ALASRU. Montevideo: Facultad de Agronomía, Universidad de la República.

Pierri, N., y Foladori, G. (2005) ¿Sustentabilidad? Desacuerdos sobre el desarrollo sustentable. Montevideo: Trabajo y Capital.

SANTos, C. (2011). ¿Qué protegen las áreas protegidas? Conservación, producción, Estado y sociedad en la implementación del Sistema Nacional de Áreas Protegidas. Montevideo: Trilce.

SAntos, C., у Сноuну, M. (2018). Los enclaves del Uruguay Natural en los márgenes del neodesarrollismo. En F. Suárez y C. Ruggerio (Comps.), Los conflictos ambientales en América Latina I: áreas de reservación, conflictos mineros e hidrocarburíferos, conflictos forestales, agronegocios. Buenos Aires: Universidad Nacional de General Sarmiento.

Sistema Nacional de Áreas Protegidas (SNAP) (2011). Plan de manejo. Paisaje protegido Quebrada de los Cuervos. Montevideo: mvotma. Recuperado de https://www.gub.uy/ministerio-ambiente/ comunicacion/publicaciones/plan-manejo-paisaje-protegido-quebrada-cuervos-sierras-del-yerbal

SNAP (2014). Plan de manejo del Parque Nacional Esteros de Farrapos e Islas del Río Uruguay. Montevideo: мvoтмa. Recuperado de https://www.gub.uy/ministerio-ambiente/comunicacion/publicaciones/ plan-manejo-del-parque-nacional-esteros-farrapos-islas-del-rio-uruguay

SNAP (2016a). Plan de manejo. Paisaje protegido Laguna de Rocha. Montevideo: мvotma. Recuperado de https://www.gub.uy/ministerio-ambiente/comunicacion/publicaciones/ plan-manejo-del-paisaje-protegido-laguna-rocha

SNAP (2016b). Plan de manejo. Paisaje protegido Valle del Lunarejo. Montevideo: мvotмa. Recuperado de https://www.gub.uy/ministerio-ambiente/comunicacion/publicaciones/ plan-manejo-paisaje-protegido-valle-del-lunarejo 
SNAP (2018). Proceso de ingreso de Paso Centurión y Sierra de Ríos al Sistema Nacional de Áreas Protegidas. Montevideo: mvotma. Recuperado de https://www.gub.uy/ministerio-ambiente/sites/ministerio-ambiente/files/documentos/publicaciones/Proyecto_ingreso_Centurion\%282\%29.pdf

Tommasino, H., y Marzaroli, J. (2008). Manual de evaluación de sistemas lecheros familiares a través de indicadores de sustentabilidad. Montevideo: First Class.

Universidad de la República, Rectorado (2010). Hacia la Reforma Universitaria. La extensión en la renovación de la enseñanza: Espacios de Formación Integral, (10). Montevideo: Universidad de la República.

Uruguay (1987, diciembre 28). Ley n. ${ }^{0}$ 15.939: Ley Forestal-Fondo Forestal-Recursos Naturales. Recuperado de https://www.impo.com.uy/bases/leyes/15939-1987.

URUguay (2000, febrero 22). Ley n. ${ }^{0}$ 17.234: Sistema Nacional de Áreas Naturales Protegidas. Recuperado de https://www.impo.com.uy/bases/leyes/17234-2000.

VAlles, H. (2000). Técnicas de observación y participación: de la observación participante a la investigación-acción-participativa. En Técnicas cualitativas de investigación social. Reflexión metodológica y práctica profesional (pp. 142-175). Madrid: Síntesis.

VAlles, H. (2002). Cuadernos metodológicos. Entrevistas cualitativas. Madrid: Centro de Investigaciones Sociológicas.

VIERA, G. (2018, mayo 4). Centurión ¿Zona protegida o confiscación? El País. Recuperado de https://www. elpais.com.uy/opinion/ecos/centurion.html. 